\title{
Significant sequelae after bacterial meningitis in Niger: a cohort study
}

\author{
Jean-François Jusot ${ }^{1 *}$, Zilahatou Tohon², Abdoul Aziz Yazi ${ }^{3}$ and Jean-Marc Collard ${ }^{4}$
}

\begin{abstract}
Background: Beside high mortality, acute bacterial meningitis may lead to a high frequency of neuropsychological sequelae. The Sahelian countries belonging to the meningitis belt experience approximately $50 \%$ of the meningitis cases occurring in the world. Studies in Africa have shown that $N$. meningitidis could cause hearing loss in up to $30 \%$ of the cases, exceeding sometimes measles. The situation is similar in Niger which experiences yearly meningitis epidemics and where rehabilitation wards are rare and hearing aids remain unaffordable. The aim of this study was to estimate the frequency of neuropsychological sequelae after acute bacterial meningitis in four of the eight regions of Niger.

Methods: Subjects exposed to acute bacterial meningitis were enrolled into a cohort with non exposed subjects matched on age and gender. Consenting subjects were interviewed during inclusion and at a control visit two months later. If clinical symptoms or psychological troubles persisted at both visits among the exposed subjects with a frequency significantly greater than that observed among the non exposed subjects, a sequelae was retained. The comparison of the frequency of sequelae between non exposed and exposed subjects to bacterial meningitis was also calculated using the Fisher exact test.

Results: Three persisting functional symptoms were registered: headaches, asthenia, and vertigo among 31.3, 36.9, and $22.4 \%$ respectively of the exposed subjects. A significant motor impairment was retrieved among $12.3 \%$ of the exposed versus $1.6 \%$ of the non exposed subjects. Hearing loss significantly disabled $31.3 \%$ of the exposed subjects and $10.4 \%$ exhibited a serious deafness.
\end{abstract}

Conclusions: This study carried out in Niger confirms two serious neurological sequelae occurring at high frequencies after bacterial meningitis: severe and profound hearing loss and motor impairment. Cochlear implantation and hearing aids are too expensive for populations living in developing countries. Neurological sequelae occurring after meningitis should sensitize African public health authorities on the development of rehabilitation centers. All these challenges can be met through existing strategies and guidelines.

Keywords: Meningococcal, Complications, Disability, Less-developed countries

\section{Background}

Beside high mortality, acute bacterial meningitis may lead to a high frequency of neuropsychological sequelae in 3 to $47 \%$ of cases, causing 160,000 yearly disabilities worldwide [1-5]. Among the three main microorganisms causing acute bacterial meningitis, Streptococcus pneumoniae (Sp) is the most lethal and most disabling followed by Haemophilus influenzae (Hi) and Neisseria meningitidis (Nm) [6].

\footnotetext{
* Correspondence: jfjusot@gmail.com

${ }^{1}$ Epidemiology/Health-Environment-Climate Unit, Centre de Recherche Médicale et Sanitaire, PO Box 10887, Niamey, Niger

Full list of author information is available at the end of the article
}

The Sahelian countries belonging to the meningitis belt experience approximately $50 \%$ of meningitis cases for a population at risk with an estimated 350 million inhabitants. Indeed, epidemics occur every year with major epidemics every $5-12$ years with high attack rates. Attack rates are high ranging from 100 to 800 per 100,000. Since the first cerebrospinal meningitis outbreak occurred in Nigeria in 1905, other epidemics have threatened the meningitis belt [7]. In 1996, large outbreaks spread across the meningitis belt leading to 250,000 suspected cases and 25,000 deaths [8]. They were mainly due to $N$. meningitidis serogroup A. In 2002 a large outbreak due

\section{Biomed Central}

(c) 2013 Jusot et al.; licensee BioMed Central Ltd. This is an Open Access article distributed under the terms of the Creative Commons Attribution License (http://creativecommons.org/licenses/by/2.0), which permits unrestricted use, distribution, and reproduction in any medium, provided the original work is properly cited. 
to $N$. meningitidis W occurred in Burkina Faso [9]. Again in 2009, 80,000 cases and more than 4,000 deaths occurred in this part of Africa [10]. Niger experienced large outbreaks in 2000, 2003 and 2009, in which $N$. meningitidis serogroup A was the main causative agent. Although a polysaccharide vaccine (A, C, W, Y serogroups of $N$. meningitidis) was widely used in mass vaccination campaigns to control transmission of the disease, a new conjugate vaccine against serogroup A was introduced in 2010. Other emerging serogroups of $N$. meningitidis, such as W135 and X, have been incriminated in recent outbreaks $[11,12]$.

In the perspective of physical rehabilitation, the most serious sequelae after acute bacterial meningitis are neurological including motor impairment, epilepsy, cecity or vision loss, speech disorder and hearing loss. In Africa, studies of bacterial meningitis sequelae have been mainly conducted more within hospital wards than in peripheral and remote health care centres usually located far from reference hospitals. The majority of the studies on post-meningitis sequelae therefore consider in-hospital outcomes whereas post-discharge outcomes are less frequently reported [4].

Hearing loss could result in irreversible neurological sequelae, frequently severe or profound, probably due to a serious or suppurative labyrinthitis occurring very early during the acute phase of meningitis and evolving towards a labyrinthitis ossificans $[13,14]$. The frequency of hearing loss exhibits large variations, between 2 and $48 \%$ depending on the studies [4]. Pneumococci have been documented as the major cause of severe/profound hearing loss leading to the majority of cochlear implantations [15]. Nevertheless, studies in Africa have shown that $N$. meningitidis could be the cause in $30 \%$ of hearing loss, sometimes exceeding measles [16-18]. The situation is similar in Niger which experiences yearly meningitis epidemics and yet where rehabilitation wards are rare and hearing aids remain unaffordable and too expensive for the disabled. In addition, frequency of sequelae after bacterial meningitis has not yet been rigorously determined in the country. Consequently, it is difficult to estimate the need for rehabilitation and sensitization for health authorities without rigorous data for decision making.

The aim of this study was therefore to estimate the frequency of the sequelae after acute bacterial meningitis in four of the eight regions of Niger.

\section{Methods}

\section{Study population}

The study population concerned inhabitants of four of the eight regions of Niger: the capital city Niamey, Dosso, Tillabery, and Maradi. These four regions comprised 9 million among the 15 million inhabitants of Niger in 2011.
From 27/12/2010 - 13/05/2011, 252 cases could be potentially enrolled in the regions targeted by this study, representing $87.3 \%$ due to $N$. meningitidis of W serogroup and $11.5 \%$ of S. pneumoniae.

\section{Study design}

The study design consisted of an exposed/non exposed cohort. Eligible subjects were retrieved after study interviewers inquired from the district health authorities if suspected meningitis cases had been notified. Identified subjects were considered exposed if one of the three main causal agents, $N$. meningitidis, S. pneumoniae or $H$. influenzae, was identified in their cerebral spinal fluid (CSF) by PCR or latex test performed as part of the routine microbiological surveillance coordinated by the CERMES. Non exposed subjects did not have any meningitis or meningeal syndrome according to the WHO suspected case classification [19]. They were selected from the family of their corresponding exposed subject and matched on gender and age. Subjects known to have suffered from a similar pathology as the post-meningitis sequelae were excluded. All the subjects were enrolled if they were 5 years old and above to facilitate the clinical and audiometric examination.

\section{Studied factors}

After enrolment, all the exposed and non exposed subjects were interviewed by two physicians, one located at Niamey and the other at Maradi. Both physicians were readily available to perform an immediate visit when a case was suspected or diagnosed in a health care facility. They were therefore aware of the infectious status of the subjects visited at the health care facility during their acute clinical phase for the exposed subjects. Non exposed subjects were visited at home. The two physicians were general practitioners trained on the aims of the study and the tools to be used (protocol, operational guide, questionnaires, audiogram, consent form). They performed a complete and in-depth clinical examination, especially focused on seeking neurological, sensitive, sensorial, and motor impairments. Clinical and psychological examinations as well as audiograms (Amplitude ${ }^{\odot}$ T-Series audiometer) were performed both at the inclusion visit and during the control visit performed 2 months later. This duration of follow-up was validated by the scientific committee of the study due to the difficulties to retrieve subjects living in remote and difficultto-access areas.

For the audiology study, the audiograms were interpreted by two physicians specialised in otorhinolaryngology who were blinded to the exposed or non exposed status of the subjects. The hearing losses were classified according to the classification of the Bureau International d'Audiophonologie. Deafness was mild, 
moderate, severe or profound when corresponding losses were from 21 to $40 \mathrm{~dB}, 41$ to $70 \mathrm{~dB}, 71$ to $90 \mathrm{~dB}$, and $>90 \mathrm{~dB}$ respectively. Severe and profound hearing losses were regrouped under serious hearing loss. Air and bone conduction was also assessed to identify the type of hearing loss.

Psychological behaviour was assessed by two psychologists during the visits with the physicians. The two psychologists belong to the technicians' category of the civil service in Niger. The following items extracted from the Conners'questionnaire were explored: blue mood, decreased energy, decreased of pep, poor motivation, serious asthenia, irritability, scared without any reason, sleeping difficulties, difficulty concentrating, nervousness, crying without any reason, and difficulty in daily living activities.

If the clinical symptoms or psychological disorders persisted at the control visit among the exposed subjects with frequencies significantly greater than those observed among the non-exposed subjects, a sequelae was retained. The audiogram result for the most altered ear at the control visit was retained to qualify the level and type of hearing loss [20].

All the findings were recorded in a questionnaire. All the questions were asked in the local language when necessary.

\section{Statistical analysis}

The statistical analysis estimated the frequency of the symptoms at inclusion between exposed and non-exposed subjects. The comparison was made using the Fisher exact test. In order to show a trend in the severity of hearing loss associated to bacterial meningitis, a trend test of Cochran-Armitrage was used. These two tests were performed using the software Winpepi [21].

A score for behavioural troubles was built and validated by estimating the reliability of 12 psychological items using the packages ltm and CMC of R software to estimate Cronbach alpha coefficient [22]. The presence of an item accounted for 1 point versus 0 in its absence. The score was calculated by summing the points with the retained items.

The comparison of the frequency of the sequelae between exposed and non exposed subjects was also calculated using the Fisher exact test. All the frequencies were estimated with a $95 \%$ confidence interval.

\section{Ethical considerations}

Study information was provided to the subjects or to their family. Written informed consent was obtained before inclusion. The study protocol was approved by the National Ethics Committee of Niger (authorisation $\left.\mathrm{N}^{\circ} 014 / / 2009 / \mathrm{CCNE}\right)$.

\section{Results}

\section{Characteristics of the subjects included}

Among the 184 subjects who met enrolment criteria, 104 suspected as clinical acute bacterial meningitis were visited and 83 were confirmed as exposed following biological confirmation of acute bacterial meningitis. Seventy nine non exposed subjects were included after one died. During the follow-up, 3 exposed subjects died (case fatality rate $=4.3 \%$ ), and 13 exposed $(15.7 \%$ ) and 14 non exposed (17.7\%) subjects were lost to follow-up two months after the inclusion visit. Finally, 67 exposed and 65 non exposed subjects were retained for the analysis to estimate the frequency of the sequelae. (Figure 1) The subjects were visited 87 days (range $=50-141$ days) on average after their inclusion visit.

\section{Symptoms associated with meningitis}

At inclusion, the 162 subjects recruited in the study consisted of 83 meningitis cases and 79 non exposed subjects. There was no gender predominance in the two groups. The mean age was 13.5 years $(\mathrm{SD}=8.9 \mathrm{y}$, range $=$ 5 - 60 years) without any difference between meningitis cases and non exposed subjects. The most frequent age groups were $5-14$ years and $15-24$ years with respectively $69.8 \%$ and $22.2 \%$ of the subjects. The predominant causal agent was $N$. meningitidis W in $86.7 \%$ of the meningitis cases; $6 \%$ were due to S. pneumoniae, $6 \%$ to N. meningitidis serogroup A and $1.2 \%$ to serogroup C (Table 1).

The main symptoms associated with meningitis are depicted in Table 2 and comprised functional symptoms like headache, asthenia, and insomnia in at least $50 \%$ of the exposed subjects. Motor impairment for tonus and walking was significantly found among $78.8 \%$ of the exposed subjects in contrast to $5.1 \%$ of non exposed subjects. Odour perception for soumbala, a well known traditional spice, was altered for $45.4 \%$ of bacterial meningitis cases. Facial muscles, bladder and anal sphincter were also impaired in respectively $17.5,31.3$ and $43.8 \%$ of the bacterial meningitis cases. No cecity was encountered, but the mean of visual acuity among meningitis cases was lower than for the non exposed subjects with respectively 8.8 and 9.3 ( $\mathrm{t}$ test $=-2.33, \mathrm{df}=128.2, \mathrm{p}=0.02$ ). Only bone conduction or both bone and air conductions and serious hearing losses were predominant during acute bacterial meningitis, with $\mathrm{OR}=4.84$ and 13.1 respectively.

Four items were validated with the Cronbach alpha coefficient (alpha $=0,847 ; 95 \% \mathrm{CI}=0,793-0,886$ ): blue mood, decreased energy, decreased of pep and severe asthenia. The exposed subjects had a score of 2.6, significantly greater than 0.8 in the non exposed subjects ( $\mathrm{t}$ test $=8.24, \mathrm{df}=147.7, \mathrm{p}<0.0001$ ). 


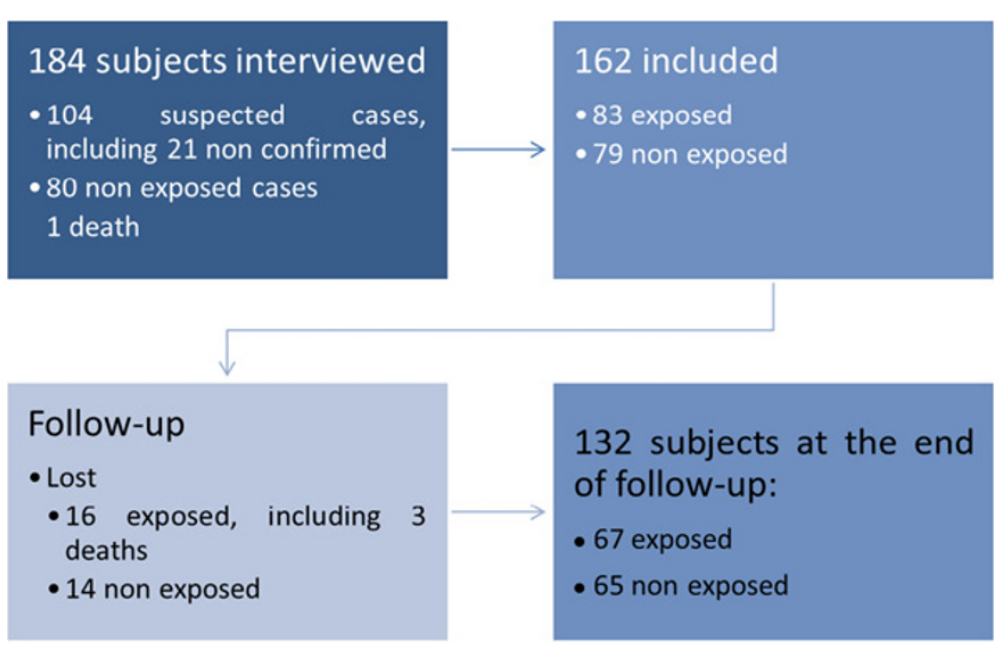

Figure 1 Flow chart explaining the distribution of the subjects during follow-up and according to their status.

\section{Sequelae associated with meningitis}

Among the 132 subjects remaining at the end of followup, there was no gender predominance with a sex ratio $\mathrm{M}: \mathrm{F}=0.94$. The mean age was 12.8 years $(\mathrm{SD}=8.1$ years, range $=5-55$ years), 13 and 12.6 years respectively for exposed and non exposed subjects.

Three functional symptoms persisted: headaches, asthenia, and vertigo among respectively $31.3,36.9$, and $22.4 \%$ of the exposed subjects with a significant risk of persistence.

The risk of motor impairment reached 7.8 among the subjects exposed to meningitis compared to the non exposed subjects. A motor impairment was significantly retrieved among $12.3 \%$ of the exposed versus $1.6 \%$ of the non exposed subjects. Hearing loss disabled $31.3 \%$ of the exposed subjects and $10.4 \%$ exhibited a serious deafness with a significant risk of 7.1. When combining motor impairment and hearing loss, the risk of neurological sequelae was 1.8 for exposed subjects versus non exposed subjects (Table 3 ).

Table 1 Basic parameters on the included subjects

\begin{tabular}{lcc}
\hline Parameter & $\begin{array}{c}\text { Exposed } \\
\mathbf{n = 8 3}\end{array}$ & $\begin{array}{c}\text { Non exposed } \\
\mathbf{n}=\mathbf{7 9}\end{array}$ \\
\hline Age: mean (SD) & $13.7(10.1)$ & $13.2(7.55)$ \\
Sex: & & \\
- Male: $\mathrm{n}(\%)$ & $44(53.0)$ & $38(48.1)$ \\
Etiological agent by PCR or Latex: & & \\
- N. meningitidis: $\mathrm{n}(\%)$ & $78(94)$ & 0 \\
$\quad$ Serogroup W135 & $72(86.7)$ & 0 \\
Location: & & $52(65.8)$ \\
- Niamey: $\mathrm{n}(\%)$ & $53(63.9)$ & \\
\hline
\end{tabular}

No behavioural troubles persisted as the score was similar among the exposed and non exposed subjects, 0.9 versus 0.5 ( $\mathrm{t}$ test $=1.75, \mathrm{df}=125.6, \mathrm{p}=0.08$ ).

\section{Discussion}

Three types of sequelae were observed in this study: functional symptoms, hearing loss, and motor impairment. We did not however address mental retardation due to the lack of standard intelligence scoring tests adapted to a country with low literacy rates. Functional symptoms like headaches and asthenia were observed at frequencies of 31.3 and $36.9 \%$. An alteration of the VIII ${ }^{\text {th }}$ pair of cranial nerve with vertigo, at least moderate and serious hearing loss was developed respectively by 22.4 , 19.4 and $10.4 \%$ of the exposed subjects. Finally, motor impairment was observed among $12.3 \%$ of exposed subjects. The frequencies of these sequelae are among the highest values retrieved in studies performed in Africa [4]. Generally, the frequency of sequelae increases with the severity of the clinical form that is usually found among pneumococcus cases. As $N$. meningitidis W was the major causal agent in our study, it raises a question on the severity linked to this serogroup which had a case fatality ratio of $12 \%$ estimated in a study performed between 2003 and 2006 in Niger [11]. Another possible explanation for the high frequencies of sequelae observed in our study is the short duration of the follow-up, three months in average, which could lead to an over estimation because some sequelae could spontaneously regress after this delay. However, increasing the study duration could lead to a higher proportion of subjects lost to follow-up and introduce a strong bias in the estimation of frequencies and risks. Nevertheless, the Société de Pathologie Infectieuse de Langue Française recommends to perform an audiogram and a neurological examination one month after acute meningitis infection, 
Table 2 Symptoms associated with meningitis at the inclusion

\begin{tabular}{|c|c|c|c|c|c|c|c|c|}
\hline \multirow[t]{2}{*}{ Symptoms } & \multicolumn{2}{|c|}{ Exposed } & \multicolumn{2}{|c|}{ Non exposed } & \multirow{2}{*}{$\begin{array}{c}\text { OR } \\
\text { Crude }\end{array}$} & \multicolumn{2}{|c|}{$95 \% \mathrm{Cl}$} & \multirow[t]{2}{*}{ p } \\
\hline & $n=83$ & $\%$ & $n=79$ & $\%$ & & Lower & Upper & \\
\hline Headaches & 79 & 59.5 & 79 & 29.1 & 3.6 & 1.8 & 7.1 & 0.0002 \\
\hline Insomnia & 80 & 58.8 & 79 & 22.8 & 4.8 & 2.3 & 10.2 & $<0.0001$ \\
\hline Convulsions before admission & 80 & 83.8 & 78 & 5.1 & 90.9 & 27.8 & 400 & $<0.0001$ \\
\hline Asthenia & 79 & 78.5 & 79 & 38.0 & 5.9 & 2.8 & 12.8 & $<0.0001$ \\
\hline Sensation to pain and to touch perturbed & 80 & 13.8 & 78 & 0 & NC & 2.7 & NC & 0.0007 \\
\hline At least one troubled reflex & 80 & 62.5 & 78 & 15.4 & 9.0 & 4.1 & 21.3 & $<0.0001$ \\
\hline Walking or tonus impaired & 80 & 78.8 & 79 & 5.1 & 66.7 & 20.8 & 286 & $<0.0001$ \\
\hline Odor of soumbala non recognised & 77 & 45.4 & 77 & 22.1 & 2.9 & 1.4 & 6.3 & 0.004 \\
\hline Masseter motility impaired & 80 & 21.3 & 78 & 0 & NC & 4.8 & NC & $<0.0001$ \\
\hline Facial motility impaired & 80 & 17.5 & 77 & 3.9 & 5.2 & 1.4 & 29.4 & 0.009 \\
\hline Trouble in the voice & 80 & 32.5 & 76 & 1.3 & 35.7 & 5.5 & 1493 & $<0.0001$ \\
\hline Vertigo & 79 & 55.7 & 79 & 15.2 & 6.9 & 3.1 & 16.4 & $<0.0001$ \\
\hline Hearing loss type & 76 & & 79 & & & & & 0.0002 \\
\hline - No & 27 & 35.5 & 53 & 67.1 & 1 & & & \\
\hline - Air conduction altered & 12 & 15.8 & 11 & 13.9 & 2.14 & 0.8 & 6.1 & 0.14 \\
\hline - Bone conduction altered or mixed & 37 & 48.7 & 15 & 19.0 & 4.84 & 2.1 & 11.2 & $<0.0001$ \\
\hline Hearing loss level & 78 & & 79 & & & & & $<0.0001$ \\
\hline - No & 27 & 34.6 & 53 & 67.1 & 1 & & & \\
\hline - Mild & 20 & 25.65 & 15 & 19.0 & 2.62 & 1.1 & 6.4 & 0.024 \\
\hline - Moderate & 11 & 14.1 & 8 & 10.1 & 2.70 & 0.9 & 8.7 & 0.067 \\
\hline - Serious & 20 & 25.65 & 3 & 3.8 & 13.1 & 3.4 & 72.9 & $<0.0001$ \\
\hline Urgent miction & 80 & 31.3 & 78 & 14.1 & 2.78 & 1.2 & 6.8 & 0.01 \\
\hline Trouble in defecation $^{+}$ & 80 & 43.8 & 78 & 14.1 & 4.8 & 2.1 & 11.4 & $<0.0001$ \\
\hline
\end{tabular}

${ }^{\dagger}$ These were constipation, faecal incontinence, faecal emergencies, and no control of the anal sphincter.

followed by a clinical surveillance of the audition every three months during one year [23].

The frequency of severe/profound hearing loss was $10.4 \%$ compared to $1.6 \%$ in a study performed in Ghana [2]. A high frequency of severe/profound hearing loss was found in a study performed in Malawi among hospitalised subjects [24]. Variations in frequencies observed in the different studies could be related to the severity of the meningitis since a severe illness could exhibit a higher risk of sequelae [25].

Persistent headaches were declared more than one week after CSF was taken and were therefore not linked to the lumbar puncture $[26,27]$. This symptom was usually found at a similar frequency in the national surveillance system of Sweden [28]. At the end of follow-up, exposed subjects had a significantly higher spontaneous declaration of headaches (27.9\%) and psychological troubles $(16.2 \%)$ than non exposed subjects $(21.5 \%$ and $3.1 \%$ respectively, data not shown). This result should incite a better assessment of functional symptoms like headaches by investigating the type, intensity, irradiation, enhancing or calming factors, and familial predisposition.
Although they were declared significantly more by the exposed subjects, psychological troubles were not considered as sequelae after assessment by the psychologists even though they were frequent at the inclusion, as shown using a reliable and validated score (Cronbach alpha coefficient $>0.8$ ). These results are in accordance with Sumpter et al. (2011) who concluded that psychological consequences are due to sequelae after meningitis and not directly due to meningitis [29].

An important asthenia persisted among $36.9 \%$ of the exposed subjects, a frequency close to the $40.1 \%$ observed in a study performed in Ghana [2]. This symptom seems to be of important interest as it is frequently found and was retained in the score, though not more frequently declared spontaneously by the exposed subjects compared to the non exposed subjects (data not shown). These discordant observations should orientate researchers' attention to the assessment of subjective psychological troubles to be probably explored more adequately using qualitative study designs [30].

One of the limits of this study could be its power which restricted the identification of other sequelae such 
Table 3 Frequency and risk of sequelae after being exposed to an acute bacterial meningitis episode

\begin{tabular}{|c|c|c|c|c|c|c|c|c|}
\hline \multirow[t]{2}{*}{ Sequelae } & \multicolumn{2}{|c|}{ Exposed } & \multicolumn{2}{|c|}{ Non exposed } & \multirow[t]{2}{*}{$p$} & \multirow[t]{2}{*}{ Crude RR } & \multicolumn{2}{|c|}{ 95\% Cl RR } \\
\hline & $\mathrm{n}=67$ & $\%$ & $n=65$ & $\%$ & & & & \\
\hline \multicolumn{9}{|l|}{ Functional symptoms } \\
\hline Headache & 67 & 31.3 & 65 & 13.8 & 0.022 & 2.3 & 1.1 & 4.6 \\
\hline Asthenia & 65 & 36.9 & 63 & 12.7 & 0.002 & 2.9 & 1.4 & 6.0 \\
\hline Vertigo & 67 & 22.4 & 65 & 7.7 & 0.027 & 2.9 & 1.1 & 7.6 \\
\hline \multicolumn{9}{|l|}{ Hearing loss assessment ${ }^{1}$} \\
\hline Bone conduction altered or mixed & 64 & 7.8 & 65 & 7.7 & 1 & 1.2 & 0.4 & 3.8 \\
\hline All levels of hearing loss & 67 & & 65 & & & & & \\
\hline - No & 46 & 68.7 & 53 & 81.5 & & & & \\
\hline - Mild & 8 & 11.9 & 8 & 12.3 & 0.8 & 1.1 & 0.5 & 2.8 \\
\hline - Moderate & 6 & 9.0 & 3 & 4.6 & 0.31 & 2.2 & 0.6 & 8.2 \\
\hline - Serious & 7 & 10.4 & 1 & 1.5 & 0.031 & 7.1 & 0.9 & 56.0 \\
\hline - Moderate/serious & 13 & 19.4 & 4 & 6.2 & 0.034 & 3.1 & 1.1 & 9.1 \\
\hline Motor impairment ${ }^{2}$ & 65 & 12.3 & 64 & 1.6 & 0.033 & 7.8 & 1 & 60.2 \\
\hline Neurological ${ }^{3}$ & 66 & 36.4 & 64 & 20.3 & 0.053 & 1.79 & 1.0 & 3.2 \\
\hline
\end{tabular}

${ }^{1}$ The most affected ear was retained.

2 Concerning walking and tonus.

${ }^{3}$ Hearing loss or motor impairment.

as epilepsy and cecity or visual loss observed in other studies $[4,26]$. Epilepsy could occur after several years of evolution whereas our study had only three months of follow-up [31]. This duration of follow-up was chosen according to the protocol study validated by the scientific committee of the study and due to difficulties encountered on the field such as health care accessibility which could inflate the percentage of participants lost to follow-up. In addition, it was difficult to collect clinical data relative to treatment because health care centres in Niger were insufficiently or not inconsistently supplied with antiepileptic drugs or corticoids; these drugs could play a role in the occurrence of sequelae after meningitis. Acute neurological signs like hydrocephalus were investigated, but none was observed. Another limit was the number of subjects lost during follow-up that could introduce a non differential bias of classification and overestimate the relative risks. A subject loss to followup of more than $15 \%$ was observed and could be explained by subjects living in remote areas with no means to come to appointments for follow-up. After taking into account lost subjects by supposing they were finally found disabled at the control visit, meningitis remained a significant risk factor only for asthenia (data not shown).

The high mortality rates due to bacterial meningitis in Niger have enhanced the surveillance and control strategies based on the implementation of reactive vaccination campaigns and early antibiotherapy. Recently, the introduction of a conjugate vaccine increased the hope of eradicating meningococci of serogroup $A$, one of the major causes of large epidemics in the meningitis belt. Nevertheless, pneumococci and meningococci of other serogroups (i.e.: $\mathrm{X}$ and $\mathrm{W}$ ) still threaten the populations of the meningitis belt. This study carried out in Niger confirms two serious neurological sequelae occurring after meningitis: high frequencies of severe and profound hearing loss, and motor impairment. Ideal followup of patients requires the most modern radiographic imageries, not affordable by Niger, for the timely detection of labyrinthitis ossificans [32]. Cochlear implantation is indicated in the first two weeks of meningitis in case of deafness [33]. Again, this treatment is not affordable to Niger's populations. Similarly, hearing aids cost 20 times higher in developing countries than when purchased elsewhere [34].

\section{Conclusions}

Neurological sequelae, particularly severe hearing loss and motor impairment, occurring after bacterial meningitis in Niger should sensitize African public health authorities towards early and rapid patient management accompanied by the development of appropriate rehabilitation measures through existing strategies and guidelines to manage hearing loss [35,36]. Continuously implementing and improving early vaccination campaigns to prevent outbreaks remains a big challenge and a way to prevent sequelae especially with the emergence of $\mathrm{W}$ in Niger and other Sahelian countries, since 2010. 


\section{Competing interests}

The authors declare that they have no competing interests.

\section{Authors' contributions}

JFJ and ZT contributed to conception and design of data. JFJ performed the analysis and interpretation of data. AAY made substantial contributions to acquisition of data and interpretation of data. JMC coordinated the microbiological surveillance in Niger. JFJ drafted the manuscript. All the authors read and approved the final manuscript and gave final approval of the version to be published.

\section{Acknowledgements}

We thank Dr. Noura Adamou who was in charge of the clinical examination of the patients; Sidikou Amadou and Hamma Ali Amadou for psychological examination; and Drs. Ganda and Morou Halidou for the interpretation of audiograms.

We are indebted to all health care workers who helped in the smooth running of this study and to the study participants, their parents as well as administrative authorities of the study areas.

We acknowledge the Biology Unit of CERMES that confirmed all the acute bacterial meningitis cases throughout the microbiological surveillance. We thank the Foreign Affairs Ministry of the French Government for its financial support (FSP No. 2005-174).

\section{Author details}

${ }^{1}$ Epidemiology/Health-Environment-Climate Unit, Centre de Recherche Médicale et Sanitaire, PO Box 10887, Niamey, Niger. ${ }^{2}$ Epidemiolgy DrPH Candidate, College of Public Health, University of Kentucky, 111 Washington Avenue, Lexington, KY 40536-0003, USA. ${ }^{3}$ Epidemiology Unit, Epicentre, PO Box 13330, Niamey, Niger. ${ }^{4}$ Biology Unit, Centre de Recherche Médicale et Sanitaire, PO Box 10887, Niamey, Niger.

Received: 17 July 2012 Accepted: 15 May 2013

Published: 21 May 2013

\section{References}

1. Tikhomirov E, Santamaria M, Esteves K: Meningococcal disease: public health burden and control. World Health Stat Q 1997, 50(3-4):170-177.

2. Hodgson A, Smith T, Gagneux S, Akumah I, Adjuik M, Pluschke G, Binka F, Genton B: Survival and sequelae of meningococcal meningitis in Ghana. Int J Epidemiol 2001, 30(6):1440-1446.

3. Oostenbrink R, Maas M, Moons KG, Moll HA: Sequelae after bacterial meningitis in childhood. Scand J Infect Dis 2002, 34(5):379-382.

4. Ramakrishnan M, Ulland AJ, Steinhardt LC, Moisi JC, Were F, Levine OS: Sequelae due to bacterial meningitis among African children: a systematic literature review. BMC Med 2009, 7:47.

5. Edmond K, Clark A, Korczak VS, Sanderson C, Griffiths UK, Rudan I: Global and regional risk of disabling sequelae from bacterial meningitis: a systematic review and meta-analysis. Lancet Infect Dis 2010, 10(5):317-328.

6. Goetghebuer T, West TE, Wermenbol V, Cadbury AL, Milligan P, Lloyd-Evans N, Adegbola RA, Mulholland EK, Greenwood BM, Weber MW: Outcome of meningitis caused by Streptococcus pneumoniae and Haemophilus influenzae type b in children in The Gambia. Trop Med Int Health 2000, 5(3):207-213.

7. Greenwood B: Manson lecture. Meningococcal meningitis in Africa. Trans R Soc Trop Med Hyg 1999, 93(4):341-353.

8. Meningococcal meningitis. http://www.who.int/mediacentre/factsheets/fs141/en

9. Decosas J, Koama JB: Chronicle of an outbreak foretold: meningococcal meningitis W135 in Burkina Faso. Lancet Infect Dis 2002, 2(12):763-765.

10. World Health Organisation: Meningitis in Chad, Niger and Nigeria: 2009 epidemic season. Wkly Epidemiol Rec 2010, 85(8):47-63.

11. Boisier P, Mainassara HB, Sidikou F, Djibo S, Kairo KK, Chanteau S: Casefatality ratio of bacterial meningitis in the African meningitis belt: we can do better. Vaccine 2007, 25(Suppl 1):A24-A29.

12. Collard JM, Maman Z, Yacouba H, Djibo S, Nicolas P, Jusot JF, Rocourt J, Maitournam R: Increase in Neisseria meningitidis serogroup W135, Niger, 2010. Emerg Infect Dis 2010, 16(9):1496-1498.

13. Merchant SN, Gopen Q: A human temporal bone study of acute bacterial meningogenic labyrinthitis. Am J Otol 1996, 17(3):375-385.
14. Vienny H, Despland PA, Lutschg J, Deonna T, Dutoit-Marco ML, Gander C: Early diagnosis and evolution of deafness in childhood bacterial meningitis: a study using brainstem auditory evoked potentials. Pediatrics 1984, 73(5):579-586.

15. Douglas SA, Sanli H, Gibson WP: Meningitis resulting in hearing loss and labyrinthitis ossificans - does the causative organism matter? Cochlear Implants Int 2008, 9(2):90-96.

16. Holborow C, Martinson F, Anger N: A study of deafness in West Africa. Int J Pediatr Otorhinolaryngol 1982, 4(2):107-132.

17. Obiako MN: Profound childhood deafness in Nigeria: a three year survey. Ear Hear 1987, 8(2):74-77.

18. Brobby GW: Causes of congenital and acquired total sensorineural hearing loss in Ghanaian children. Trop Doct 1988, 18(1):30-32.

19. Bacterial meningitis (including Haemophilus influenzae type b (Hib), Neisseria meningitidis, and Streptococcus pneumoniae). http://www.who.int/ immunization_monitoring/diseases/meningitis_surveillance/en/index.html.

20. Wait JW, Stanton L, Schoeman JF: Tuberculosis meningitis and attention deficit hyperactivity disorder in children. J Trop Pediatr 2002, 48(5):294-299.

21. Abramson JH: WINPEPI updated: computer programs for epidemiologists, and their teaching potential. Epidemiol Perspect Innov 2011, 8(1):1.

22. Moret L, Mesbah M, Chwalow J, Lellouch J: Internal validation of a measurement scale: relation between principal component analysis, Cronbach's alpha coefficient and intra-class correlation coefficient. Rev Epidemiol Sante Publique 1993, 41(2):179-186.

23. Société de Pathologie Infectieuse de Langue Française; Collège des Universitaires des Maladies Infectieuses et Tropicales (CMIT); Association Pédagogique Nationale pour l'Enseignement de la Thérapeutique (APNET); Société Française de Microbiologie (SFM); Société Française de Médecine d'Urgence (SFMU); Société Française de Neurologie (SFN); Société Française d'ORL (SFORL); Société Française de Pédiatrie (SFP); Société Nationale Française de Médecine Interne (SNFMI); Société de Réanimation de Langue Française (SRLF): Management of acute community-acquired bacterial meningitides, except in newborn infants. Short text. November 2008. Société de pathologie infectieuse de langue française. Rev Neurol (Paris) 2009, 165:F205-F216.

24. Forsyth H, Kalumbi F, Mphaka E, Tembo M, Mwenechanya J, Kayira K, Bwanaisa L, Njobvu A, Walsh A, Molyneux E: Hearing loss in Malawian children after bacterial meningitis: incidence and risk factors. Audiological Medicine 2004, 2(2):100-107.

25. Davey PG, Jabeen FJ, Harpur ES, Shenoi PM, Geddes AM: A controlled study of the reliability of pure tone audiometry for the detection of gentamicin auditory toxicity. J Laryngol Otol 1983, 97(1):27-36.

26. Kuntz KM, Kokmen E, Stevens JC, Miller P, Offord KP, Ho MM: Post-lumbar puncture headaches: experience in 501 consecutive procedures. Neurology 1992, 42(10):1884-1887.

27. Lowery S, Oliver A: Incidence of postdural puncture headache and backache following diagnostic/therapeutic lumbar puncture using a $22 \mathrm{G}$ cutting spinal needle, and after introduction of a $25 \mathrm{G}$ pencil point spinal needle. Paediatr Anaesth 2008, 18(3):230-234.

28. Berg S, Trollfors B, Hugosson S, Fernell E, Svensson E: Long-term follow-up of children with bacterial meningitis with emphasis on behavioural characteristics. Eur J Pediatr 2002, 161(6):330-336.

29. Sumpter R, Brunklaus A, McWilliam R, Dorris L: Health-related quality-of-life and behavioural outcome in survivors of childhood meningitis. Brain Inj 2011, 25(13-14):1288-1295.

30. Greenhalgh T, Taylor R: Papers that go beyond numbers (qualitative research). BMJ 1997, 315:740-743.

31. Salih MA, Khaleefa OH, Bushara M, Taha ZB, Musa ZA, Kamil I, Hofvander Y, Olcen P: Long term sequelae of childhood acute bacterial meningitis in a developing country. A study from the Sudan. Scand J Infect Dis 1991, 23(2):175-182

32. Isaacson B, Booth T, Kutz JW Jr, Lee KH, Roland PS: Labyrinthitis ossificans: how accurate is MRI in predicting cochlear obstruction? Otolaryngol Head Neck Surg 2009, 140(5):692-696.

33. Tinling SP, Colton J, Brodie HA: Location and timing of initial osteoid deposition in postmeningitic labyrinthitis ossificans determined by multiple fluorescent labels. Laryngoscope 2004, 114(4):675-680.

34. Kumar S: WHO tackles hearing disabilities in developing world. Lancet 2001, 358(9277):219. 
35. Lasisi OA, Ayodele JK, ljaduola GT: Challenges in management of childhood sensorineural hearing loss in sub-Saharan Africa, Nigeria. Int J Pediatr Otorhinolaryngol 2006, 70(4):625-629.

36. Tucci $\mathrm{D}$, Merson MH, Wilson BS: A summary of the literature on global hearing impairment: current status and priorities for action. Otol Neurotol 2010, 31(1):31-41.

doi:10.1186/1471-2334-13-228

Cite this article as: Jusot et al: Significant sequelae after bacterial meningitis in Niger: a cohort study. BMC Infectious Diseases 2013 13:228.

\section{Submit your next manuscript to BioMed Central and take full advantage of:}

- Convenient online submission

- Thorough peer review

- No space constraints or color figure charges

- Immediate publication on acceptance

- Inclusion in PubMed, CAS, Scopus and Google Scholar

- Research which is freely available for redistribution 